\title{
A dynamical model for quantum memory channels
}

\author{
Vittorio Giovannetti \\ NEST-INFM \& Scuola Normale Superiore, I-56126 Pisa, Italy.
}

(Dated: November 13, 2018)

\begin{abstract}
A dynamical model for quantum channel is introduced which allows one to pass continuously from the memoryless case to the case in which memory effects are present. The quantum and classical communication rates of the model are defined and explicit expression are provided in some limiting case. In this context we introduce noise attenuation strategies where part of the signals are sacrificed to modify the channel environment. The case of qubit channel with phase damping noise is analyzed in details.
\end{abstract}

PACS numbers: 03.67.Hk, 03.65.Ud, 89.70.+c

\section{INTRODUCTION}

In memoryless quantum channels successive signals (channel uses) are affected by independent, uniform sources of noise 1, 2, 3, 4, 5]. On the other hand, memory channels are characterized by the presence of correlated source of noise where each channel use is directly or indirectly affected by the previous ones. Preliminary results in the study of such systems has been obtained in Ref. 6] where it was pointed out that entangled codes can be useful in achieving optimal channel performances. Subsequently some of these results have been generalized to the continuous variable case in Refs. 7, 8], while a systematic analysis of the problem has been proposed in Refs. 9, [10]. In this paper we present a "dynamical" model for studying memory effects in quantum communication where the noise correlations are derived from the interactions between the transmitted signals and the channel environment. By varying the time intervals at which signals are produced by the sender of the message, the model simulates different communication scenarios. Memoryless configurations for instance are recovered as a limiting case in which the signals are transmitted at a frequency much lower than the inverse of the characteristic time of the channel environment relaxation. In this context we introduce also noise attenuation protocols where the sender alternates sequences of carrying-messages signals with sequences of signals which are employed to modify the environment response but which do not carry any messages to the receiver. Since timescales are fundamental in our model, we characterize its efficiency by introducing the transmission rates of the communication line. These are dimensional quantities (of dimension equal to an inverse time) which measure the maximal number of qubits or bits of information that can be transferred reliably (i.e. with unit fidelity) through the channel per unit of transmitting time. Transmission rates are peculiar of our model as previous works [ 6, 7, 8, 9, 10] were concerned in characterizing memory channels in terms of information capacities, i.e. the maximum number of qubits (or bits) that can be reliably transferred through the channel per channel uses. These figures of merit (i.e. rates and capacities) are in general distinct, but are proportional to each other when the sender of the message encodes her/his messages in regular sequence of signals (see Sec. IVA).

In Sec. II we introduce the channel model by focusing on the physical assumption which underline its definition. In Sec. III we discuss the memory effects present in the system and we introduce the noise attenuation protocols. In Sec. IV and Sec. D we define the transmission rates of the channel and we compute their values in some extremal case. Finally in Sec.VI an example of a dephasing qubit channel with memory is discussed.

\section{THE MODEL}

Consider a communication line where messages are encoded into some internal degree of freedom (e.g. polarization, spin etc.) of a collection of identical physical objects $\mathrm{C}_{1}, \mathrm{C}_{2}, \cdots$ which propagate through the medium $\mathrm{E}$ that separates the sender (say Alice) from the receiver (Bob). The $\mathrm{C}_{j}$ are the information carriers of the system: they are locally produced by Alice and organized in a time-ordered sequence $s=\left\{\tau_{1}, \tau_{2}, \cdots\right\}$ with $\tau_{j}>0$ being the time interval between the instants $t_{j+1}$ and $t_{j}$ at which $\mathrm{C}_{j+1}$ and $\mathrm{C}_{j}$ enter $\mathrm{E}$ respectively. We will assume the effective transit time $\mathcal{T}_{t r}$ it takes for the carriers for reaching Bob to be constant and shorter than the intervals $\tau_{j}$ at which they are injected into the medium (fast propagation condition). The first condition guarantees that the time-ordering of $s$ is preserved in the propagation (i.e. Bob will receive the $(j+1)$-th carrier only after a time $\tau_{j}$ from the arrival of the $j$-th carrier). The second condition instead guarantees that $\mathrm{E}$ interacts only with one carrier at a time. Therefore, if $R$ is the density matrix of the carriers at Alice location, after a time $\mathcal{T}_{t r}$ Bob will receive the state

$$
R^{\prime}=\operatorname{Tr}_{E}\left\{W\left(R \otimes \rho_{0}\right) W^{\dagger}\right\}
$$

where $\rho_{0}$ is the initial state of $\mathrm{E}$, and where

$$
W=\cdots V_{j} U_{j} \cdots V_{2} U_{2} V_{1} U_{1}
$$

is the unitary operator which describes the coupling between the internal degree of freedom of the carriers and E. 


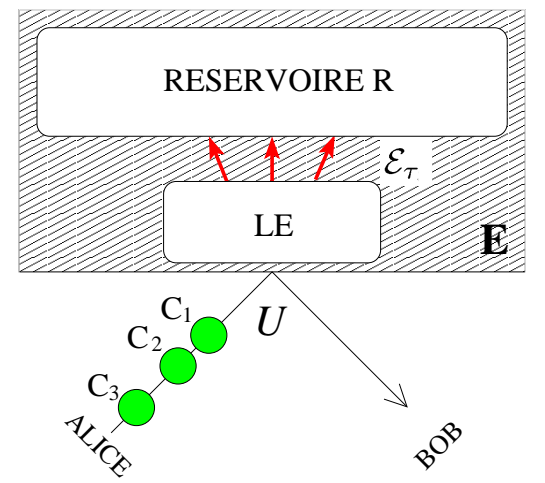

FIG. 1: Schematic of the communication scenario. Alice encodes her messages in the internal degree of freedom of the carriers $\mathrm{C}_{1}, \mathrm{C}_{2}, \cdots$, which propagates in a time-ordered sequence toward Bob. The carriers interact one at a time with the local environment LE, while LE undergoes a dissipative evolution through its interaction with the reservoir $\mathrm{R}$.

In Eq. (2) the terms

$$
U_{j} \equiv T \exp \left\{-\frac{i}{\hbar} \int_{t_{j}}^{t_{j}+\mathcal{T}_{t r}} d t\left[H_{C_{j} E}(t)+H_{E}\right]\right\},
$$

describe the interaction between $\mathrm{C}_{j}$ and $\mathrm{E}$ (here $H_{C_{j} E}(t)$ is the effective time dependent Hamiltonian that couples $\mathrm{C}_{j}$ and $\mathrm{E}$, while $H_{E}$ is the free Hamiltonian of the medium). Working in a strong coupling regime we will neglect the contribution of $H_{E}$ in Eq. (3) and we will assume the $U_{j}$ to be uniform with respect to the label $j$. On the other hand, the terms $V_{j}$ of Eq. (2) describe the free evolution of $\mathrm{E}$ in the time interval between the instant $t_{j}+\mathcal{T}_{t r}$ when $\mathrm{C}_{j}$ leaves the environment and the instant $t_{j+1}$ when $\mathrm{C}_{j+1}$ enters it, i.e.

$$
V_{j} \equiv \exp \left\{-\frac{i}{\hbar} H_{E}\left(\tau_{j}-\mathcal{T}_{t r}\right)\right\} \simeq \exp \left\{-\frac{i}{\hbar} H_{E} \tau_{j}\right\}
$$

In the following we identify two distinct components of the medium E: a finite dimensional Local Environment (LE) component which is directly coupled with the carriers through the $U_{j}$, and a huge Reservoir (R) component which is coupled with LE but not with the carriers (see Fig. 1). The free evolution (4) is supposed to induce a dissipative dynamics which transforms any initial states of LE into a stationary configuration $\sigma_{0}$, with $\tau_{E}$ being the characteristic time of the process. This is equivalent 11] to introducing a one-parameter family $\mathcal{F} \equiv\left\{\mathcal{E}_{\tau}\right\}_{\tau \geqslant 0}$ of Completely Positive, Trace preserving (CPT) which, given $\sigma$ the initial state of LE at some time $t_{0}$, represents its evolution at time $t_{0}+\tau$ with the density matrix $\mathcal{E}_{\tau}(\sigma)$. In this formalism $\mathcal{E}_{0}$ coincides with identity map on $\mathcal{H}_{L E}$. On the other hand the stationary state $\sigma_{0}$ of LE is defined by the property

$$
\mathcal{E}_{\tau}\left(\sigma_{0}\right)=\sigma_{0} \text { for all } \tau \geqslant 0,
$$

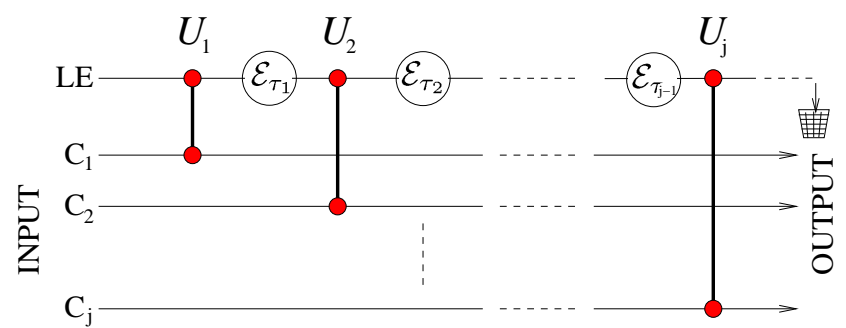

FIG. 2: Circuit representation of Eq. (7). The local environment LE interacts through the unitary couplings $U_{j}$ (represented by the small red circles in the figure) with one carriers at a time. Between two consecutive interactions with the carriers instead LE undergoes the dissipative evolution described by the transformations $\mathcal{E}_{\tau_{j}}$ (open circles).

while the characteristic time $\tau_{E}$ by the property

$$
\mathcal{E}_{\tau \geqslant \tau_{E}}(\Theta)=\sigma_{0} \operatorname{Tr} \Theta,
$$

for all bounded operator $\Theta$ of $\mathcal{H}_{L E}$. An example of $\mathcal{F}$ satisfying the above conditions will be presented in Sec. DI

Under the above approximations Eq. (1) provides a bouncing ball description of the carrier-environment interactions where the carriers-balls move toward the LE-wall according to the time-ordered sequence $s=\left\{\tau_{1}, \tau_{2}, \cdots\right\}$ chosen by the "pitcher" Alice and "hit" instantaneously the local environment LE one at a time (see Fig. 1). The resulting transformation is a time ordered product of interactions $U_{j}$ and relaxation processes $\mathcal{E}_{\tau_{j}}$ (see Fig. 2). Assuming LE to be initially in the stationary state $\sigma_{0}$ this gives

$$
\begin{aligned}
R^{\prime}=\operatorname{Tr}_{L E}\{ & \cdots \circ \mathcal{E}_{\tau_{j}} \circ \mathcal{U}_{j} \circ \cdots \\
& \left.\circ \mathcal{E}_{\tau_{2}} \circ \mathcal{U}_{2} \circ \mathcal{E}_{\tau_{1}} \circ \mathcal{U}_{1}\left(R \otimes \sigma_{0}\right)\right\},
\end{aligned}
$$

where the partial trace is performed on $\mathcal{H}_{L E}, \mathcal{U}_{j}(\cdots)$ stands for the unitary mapping $U_{j}(\cdots) U_{j}^{\dagger}$ on $\mathcal{H}_{C_{j}} \otimes \mathcal{H}_{L E}$, and "o" indicates the composition of super-operators. It is important to note that in our model each sequence $s=$ $\left\{\tau_{1}, \tau_{2}, \cdots\right\}$ is characterized by a distinct input-output relation (7).

\section{MEMORY EFFECTS}

Here we give an overview of the memory effects which are accounted for by the model introduced in Sec. [II

Because of the time ordering of Eq. (77) the output state of a carrier might depend on the input state of the carriers which precedes it in $s$ but it is always independent from the input state of the carriers which follows it in the sequence. As a matter of fact Eq. (7) closely resembles the memory channels analyzed by Kretschmann and Werner in Ref. 10]. To make this more explicit we rewrite this equation in terms of of the discrete family of 
CPT maps $\left\{\Phi_{s}^{(n)}\right\}_{n}$ where

$$
\begin{aligned}
\Phi_{s}^{(n)}(R) \equiv \operatorname{Tr}_{L E}\left\{\mathcal{U}_{n} \circ \mathcal{E}_{\tau_{n-1}} \circ \mathcal{U}_{n-1}\right. \\
\left.\circ \cdots \circ \mathcal{E}_{\tau_{1}} \circ \mathcal{U}_{1}\left(R \otimes \sigma_{0}\right)\right\},
\end{aligned}
$$

is the output state (7) corresponding to the density matrix $R$ of $\otimes_{j=1}^{n} \mathcal{H}_{C_{j}}$ associated with the first $n$ carriers of the sequence $s$ (here $\mathcal{H}_{C_{j}}$ is the Hilbert space associated with the internal degree of freedom of the $j$-th carrier). Therefore the model of Sec. I originates proper memory effects analogous to those of Refs. [6, 8, 9, 10] but avoids the feed-forward correlations of Ref. [7]. For instance Markovian correlated noise can be recovered by properly

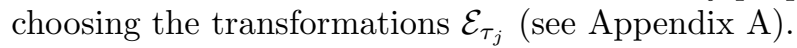

\section{A. Memoryless configuration}

Assume Alice is producing a sequence $s$ with intervals $\tau_{j}$ greater than or equal to the characteristic relaxation time $\tau_{E}$ of the dissipation process $\mathcal{F}$ - see part a) of Fig. [3 In this case, after each interaction, the local environment LE has enough time to relax into the stationary configuration $\sigma_{0}$ before a new carrier begins interacting with it. Under this hypothesis Eqs. (6) and (8) yield

$$
\Phi_{s}^{(n)}=\mathcal{N}^{\otimes n}
$$

where $\mathcal{N}$ is the CPT map which transforms the density matrices $\rho$ of a single carrier into

$$
\mathcal{N}(\rho)=\operatorname{Tr}_{L E}\left\{\mathcal{U}\left(\rho \otimes \sigma_{0}\right)\right\} .
$$

Equation (9) describes a memoryless configuration where the noise acts on the $\mathrm{C}_{j}$ independently.

\section{B. Generalized memoryless configuration}

A generalization of (9) is obtained when the carriers are organized in identical independent groups of $m$ elements each. Here it is convenient to express the elements of $s$ as $\tau_{g, \ell}$ where $g=1,2, \cdots$ is the group index, while $\ell \in\{1, \cdots, m\}$ labels the carriers within a given group. In this notation the time interval

$$
T_{g}=\sum_{\ell=1}^{m-1} \tau_{g, \ell}
$$

gives the "length" of the $g$-th group while $\Delta T_{g}=\tau_{g, m}$ is the interval which separates the last element of the $g$ th group from the first element of the $(g+1)$-th group. We do not assume any restrictions on the time intervals $\left\{\tau_{g, \ell}\right\}_{\ell=1, \cdots, m-1}$ which separates carriers belonging to the same group but we require carriers of distinct subgroups to be separated by time intervals larger than $\tau_{E}$, i.e. $\Delta T_{g} \geqslant \tau_{E}$ - see Fig. 3 part b). In this case from

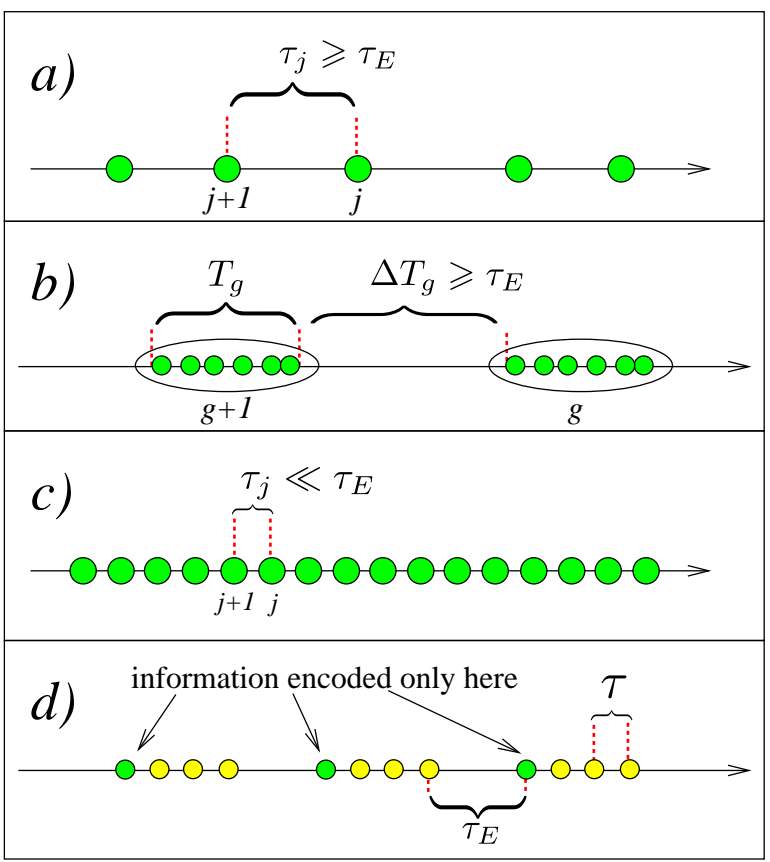

FIG. 3: Some relevant configurations. Part a): memoryless configuration (9). The carriers (represented by the green circles) are separated by time intervals $\tau_{j}$ which are greater than the dissipation time $\tau_{E}$ of the local environment. Part $b$ ): generalized memoryless configuration (12). Here the carriers are divided in groups labeled by the index $g$. The groups are separated by time intervals $\Delta T_{g}$ which are greater than the dissipation time $\tau_{E}$. Part $c$ ): perfect memory channel (14). Here the distance between two consecutive carriers is negligible with respect to $\tau_{E}$ inhibiting the relaxation of LE. Part $d$ ): example of a noise attenuation protocol. Alice sends uniform sequences of signals composed by $n$ carriers (the B carriers of the protocol represented by yellow circles in the picture) which have been prepared in the same input state $\rho_{0}$ and which are separated by time intervals $\tau$. These carriers do not convey any message to Bob and are employed only to "program" the environment response. The information is instead encoded into the $(n+1)$-th carrier (the A carriers of the protocol represented by the green circles). The sequence repeats after a time interval $\tau_{E}$ to allow LE to return to the stationary configuration.

Eq. (8) follows that the transformation of the carriers of the first $G$ groups can be expressed as

$$
\Phi_{s}^{(n)}=\otimes_{g=1}^{G} \mathcal{M}_{s}^{(g)},
$$

where $n=m G$ and

$$
\begin{aligned}
& \mathcal{M}_{s}^{(g)}(\rho) \equiv \\
& \operatorname{Tr}_{L E}\left\{\mathcal{U}_{g, m} \circ \mathcal{E}_{\tau_{g, m-1}} \circ \cdots \circ \mathcal{E}_{\tau_{g, 1}} \circ \mathcal{U}_{g, 1}\left(\rho \otimes \sigma_{0}\right)\right\},
\end{aligned}
$$

is the CPT map associated with the $m$ carriers $\mathrm{C}_{g, 1}$, $\cdots, \mathrm{C}_{g, m}$ of the $g$-th group. By comparison with Eq. (9), Eq. (12) describes a memoryless channel where the groups are the effective information carriers of the model. In particular if the sets $\left\{\tau_{g, \ell}\right\}_{\ell=1, \cdots, m}$ are uniform 
with respect to the group label $g$, one has $\mathcal{M}_{s}^{(g)}=\mathcal{M}_{s}^{\left(g^{\prime}\right)}$ for all $g$ and $g^{\prime}$ and the transformation (12) has once again the standard tensor structure $\mathcal{M}_{s}^{\otimes G}$.

\section{Perfect memory channel}

Consider the case where $\tau_{j} \ll \tau_{E}$ for all $j$. In this limit the local environment relaxation process is inhibited by the frequent interactions with the carriers. Consequently the $\mathcal{E}_{\tau_{j}}$ are replaced by the identity transformation on $\mathcal{H}_{L E}$ and Eq. (8) yields

$$
\Phi_{s}^{(n)}(R)=\operatorname{Tr}_{L E}\left\{\mathcal{U}_{n} \circ \cdots \circ \mathcal{U}_{2} \circ \mathcal{U}_{1}\left(R \otimes \sigma_{0}\right)\right\} .
$$

This expression describes a perfect memory channel [9, 10] where the information transferred from the carriers to the finite dimensional local environment LE is not dissipated into the reservoir $\mathrm{R}$ of Fig. 11. These maps are asymptotically equivalent [10] to noiseless channel where each carriers can transfer $\log _{2} D$ qubits of quantum information reliably (here $D$ is the dimension of the Hilbert space $\mathcal{H}_{C}$ of a single carrier).

\section{Noise attenuation protocols}

Here we present a communication strategy which explicitly exploits the fact that in our model the environment is effected by the signaling process. In this protocols only a subset A of the transmitted carriers is used to encode messages to Bob. The remaining carriers (subset B) are instead employed for perturbing LE in such a way that the $\mathrm{C}_{j}$ on which the messages are encoded have a better chance to reach Bob without being corrupted. In other words the B carriers are used by the sender as control parameters to program the environment response to the A carriers. A simple implementation of a noise attenuation scheme is shown in Fig. 3 part $d$ ). Here the $\mathrm{B}$ carriers are composed by uniform strings of $n$ states $\rho_{0}$ (represented by the yellow circles) separated by equal time intervals $\tau$. The information is instead encoded a single carrier (green circles) and the whole structure repeats after a relaxation time $\tau_{E}$ - this last assumption is not fundamental but allows us to treat the input-output relations of the $\mathrm{A}$ carriers as a memoryless channel of the form (9). In this configuration the transformation of the A carriers which comes from solving Eq. (17) can be computed as follows. First we determine the modified state $\sigma_{n}$ of LE which arises from the interactions with the $\mathrm{B}$ carriers. This is accomplished by solving the set of coupled equations analogous to those of Ref. [12],

$$
\left\{\begin{array}{l}
\sigma_{j}^{\prime}=\operatorname{Tr}_{C}\left\{\mathcal{U}\left(\rho_{0} \otimes \sigma_{j}\right)\right\} \\
\sigma_{j+1}=\mathcal{E}_{\tau}\left(\sigma_{j}^{\prime}\right)
\end{array}\right.
$$

where the trace is performed over the carrier degree of freedom, $\mathcal{U}$ is the usual carrier-LE coupling superoperator and $j=0,1, \cdots, n-1$. The density matrix $\sigma_{n}$ which results from (15) is then used to determine the output state of the A carriers according to the equation

$$
\overline{\mathcal{N}}(\rho) \equiv \operatorname{Tr}_{L E}\left\{\mathcal{U}\left(\rho \otimes \sigma_{n}\right)\right\}
$$

The transformation (16) is in general different from Eq. (10) and depends explicitly on the parameters $n$, $\tau$ and $\rho_{0}$ that are controlled by Alice. The basic idea of a noise attenuation scheme is to appropriately select such parameters in order to get a transformed mapping $\overline{\mathcal{N}}$ which is less noisy than the original mapping $\mathcal{N}$. An example of this effect will be presented in Sec. VI

\section{TRANSMISSION RATE OF A SEQUENCE}

Timescales play a fundamental role in the model presented in Sec. [II. Therefore a proper way to characterize it, is by introducing its quantum and classical transmission rates. In simple terms these quantities measure, respectively, the maximum number of qubits and bits per second that Alice can encode into the carriers sequence $s$ without compromising the readability of the transmitted messages. The formal definition of the rate of the sequence $s$ is constructed as follows.

First of all we introduce the discrete value function $n_{s}(T)$ which, given the sequence $s$, counts the number of carriers which fit [13] in the time interval [0, T[. Furthermore, for any $\epsilon>0$ and $T>0$ we define $q_{s}(\epsilon, T)$ to be the dimension -in qubits units- of the largest Hilbert sub-space of $\mathcal{H}(T) \equiv \otimes_{j=1}^{n_{s}(T)} \mathcal{H}_{C_{j}}$ which allows for a fidelity of the transmitted state greater than $1-\epsilon$. This is

$$
\begin{aligned}
q_{s}(\epsilon, T) & =\max _{d}\left\{\log _{2} d: \exists \mathcal{H}_{\text {code }} \operatorname{dim} \mathcal{H}_{\text {code }}=d, \exists \mathcal{A}, \mathcal{D}\right. \\
& \left.\forall|\Psi\rangle \in \mathcal{H}_{\text {code }} F\left(\Psi, \mathcal{D} \circ \Phi_{s}^{(T)} \circ \mathcal{A}\right)>1-\epsilon\right\},
\end{aligned}
$$

where $\mathcal{H}_{\text {code }}$ are Hilbert sub-spaces of $\mathcal{H}(T), \mathcal{A}$ and $\mathcal{D}$ are encoding and decoding CPT maps on $\mathcal{H}(T)$ applied, respecitively, by Alice and Bob to the carriers, and

$$
F\left(\Psi, \mathcal{D} \circ \Phi_{s}^{(T)} \circ \mathcal{A}\right) \equiv\left\langle\Psi\left|\mathcal{D} \circ \Phi_{s}^{(T)} \circ \mathcal{A}(|\Psi\rangle\langle\Psi|)\right| \Psi\right\rangle,
$$

is the fidelity between the input state $|\Psi\rangle \in \mathcal{H}_{\text {code }}$ and the decoded output state $\mathcal{D} \circ \Phi_{s}^{(T)} \circ \mathcal{A}(|\Psi\rangle\langle\Psi|$ ) (for easy of notation $\Phi_{s}^{(T)}$ indicates the map $\Phi_{s}^{\left(n_{s}(T)\right)}$ of Eq. (8) that acts on the $n_{s}(T)$ carriers of $s$ which lie on $[0, T[)$. The quantum transmission rate $r_{q}(s)$ of $s$ is thus given by the ratio $q_{s}(\epsilon, T) / T$ in the the limits $\epsilon \rightarrow 0, T \rightarrow \infty$, i.e. [14]

$$
r_{q}(s)=\lim _{\epsilon \rightarrow 0} \limsup _{T \rightarrow \infty} \frac{q_{s}(\epsilon, T)}{T} .
$$

Analogously we define the classical transmission rate $r_{c}(s)$ of $s$ by substituting the function $q_{s}(\epsilon, T)$ with the largest number of classical distinguishable messages 
$c_{s}(\epsilon, T)$ that can be transmitted to Bob with fidelity greater than $1-\epsilon$, i.e.

$$
r_{c}(s)=\lim _{\epsilon \rightarrow 0} \limsup _{T \rightarrow \infty} \frac{c_{s}(\epsilon, T)}{T},
$$

where as in Eq. (17) one has

$$
\begin{array}{r}
c_{s}(\epsilon, T)=\max _{d}\left\{\log _{2} d: \exists \mathcal{H}_{\text {code }} \operatorname{dim} \mathcal{H}_{\text {code }}=d, \exists \mathcal{A}, \mathcal{D}\right. \\
\left.\forall k \in\{1, \cdots, d\} \quad F\left(\Psi_{k}, \mathcal{D} \circ \Phi_{s}^{(T)} \circ \mathcal{A}\right)>1-\epsilon\right\}(21)
\end{array}
$$

with $\left|\Psi_{1}\right\rangle,\left|\Psi_{2}\right\rangle, \cdots,\left|\Psi_{d}\right\rangle$ being an orthonormal basis of $\mathcal{H}_{\text {code }}$.

\section{A. Upper and lower bounds}

A simple upper bound for the quantum rate $r_{q}(s)$ of $s$ can be derived from Eq. (19) as follows, 15]

$$
\begin{aligned}
r_{q}(s) & =\lim _{\epsilon \rightarrow 0} \limsup _{T \rightarrow \infty} \frac{n_{s}(T)}{T} \frac{q_{s}(\epsilon, T)}{n_{s}(T)} \\
& \leqslant\left[\lim _{\epsilon \rightarrow 0} \limsup _{T \rightarrow \infty} \frac{q_{s}(\epsilon, T)}{n_{s}(T)}\right] \limsup _{T^{\prime} \rightarrow \infty} \frac{n_{s}\left(T^{\prime}\right)}{T^{\prime}} \\
& =Q_{s} / \tau_{s}^{\prime},
\end{aligned}
$$

where $\tau_{s}^{\prime}$ is the minimum average first-neighbors distance among the carriers of $s$ defined by

$$
1 / \tau_{s}^{\prime}=\limsup _{T^{\prime} \rightarrow \infty} \frac{n_{s}\left(T^{\prime}\right)}{T^{\prime}}=\lim _{T^{\prime} \rightarrow \infty} \sup _{t \geqslant T^{\prime}} \frac{n_{s}(t)}{t} .
$$

On the other hand

$$
Q_{s}=\lim _{\epsilon \rightarrow 0} \limsup _{T \rightarrow \infty} \frac{q_{s}(\epsilon, T)}{n_{s}(T)}=\lim _{\epsilon \rightarrow 0} \limsup _{n \rightarrow \infty} \frac{q_{s}(\epsilon, n)}{n}
$$

defines the quantum capacity [2, 10, 16, 17] associated with the maps $\left\{\Phi_{s}^{(n)}\right\}_{n}$ of Eq. (8) (in this expression $q_{s}(\epsilon, n)$ is given by (17) with $n_{s}(T)$ replaced by $n$ ).

A lower bound for $r_{q}(s)$ is instead obtained as follows 15

$$
\begin{aligned}
r_{q}(s) & =\lim _{\epsilon \rightarrow 0} \limsup _{T \rightarrow \infty} \frac{n_{s}(T)}{T} \frac{q_{s}(\epsilon, T)}{n_{s}(T)} \\
& \geqslant\left[\lim _{\epsilon \rightarrow 0} \limsup _{T \rightarrow \infty} \frac{q_{s}(\epsilon, T)}{n_{s}(T)}\right] \liminf _{T^{\prime} \rightarrow \infty} \frac{n_{s}\left(T^{\prime}\right)}{T^{\prime}} \\
& =Q_{s} / \tau_{s}^{\prime \prime},
\end{aligned}
$$

where $\tau_{s}^{\prime \prime} \geqslant \tau_{s}^{\prime}$ is the maximum first-neighbors average distance among the carriers of $s$ defined by

$$
1 / \tau_{s}^{\prime \prime}=\liminf _{T^{\prime} \rightarrow \infty} \frac{n_{s}\left(T^{\prime}\right)}{T^{\prime}}=\lim _{T^{\prime} \rightarrow \infty} \inf _{t \geqslant T^{\prime}} \frac{n_{s}(t)}{t} .
$$

If the sequences $s$ is such that $\lim _{T \rightarrow \infty} n_{s}(T) / T=1 / \tau_{s}$ exists, one has $\tau_{s}^{\prime}=\tau_{s}^{\prime \prime}=\tau_{s}$ with $\tau_{s}$ being the average first-neighbors distance among the carriers. These are the regular sequences of the model: for them Eqs. (22) and (25) coincide and the transmission rate is proportional to the quantum capacity of the channel, i.e.

$$
r_{q}(s)=Q_{s} / \tau_{s}
$$

The same analysis can be repeated also for the classical rate $r_{c}(s)$ of Eq. (20). In particular, in this case, Eqs. (22), (25) and (27) still apply by replacing $Q_{s}$ with the classical capacity $C_{s}$ of the maps $\left\{\Phi_{s}^{(n)}\right\}_{n}$ defined by

$$
C_{s}=\lim _{\epsilon \rightarrow 0} \limsup _{n \rightarrow \infty} \frac{c_{s}(\epsilon, n)}{n} \text {. }
$$

\section{B. Some solvable configurations}

The maximizations implicit in Eqs. (24) and (28) are in general difficult to solve. However, following the analysis of Refs. 10, 16] one can bound the capacities $Q_{s}$ and $C_{s}$ by means of the coherent information [18] and of the Holevo information [19] of $\Phi_{s}^{(n)}$, respectively. In particular we have

$$
Q_{s} \leqslant \limsup _{N \rightarrow \infty} \max _{R} \frac{J\left(\Phi_{s}^{(N)}, R\right)}{N}
$$

where the maximization is performed over all density matrices $R$ of $N$ carriers and

$$
J\left(\Phi_{s}^{(N)}, R\right) \equiv S\left(\Phi_{s}^{(N)}(R)\right)-S\left(\left(\Phi_{s}^{(N)} \otimes \mathcal{I}_{A}\right)\left(\Psi_{R}\right)\right),
$$

is the coherent information [18] of $\Phi_{s}^{(N)}(R)$. In the above expression $S(R)=-\operatorname{Tr}\left[R \log _{2} R\right]$ is the von Neumann entropy, $\Psi_{R}$ is a generic purification of $R$ constructed by adding an ancillary Hilbert space $\mathcal{H}_{A}$, and $\mathcal{I}_{A}$ is the identical map on $\mathcal{H}_{A}$. Analogously one has

$$
C_{s} \leqslant \limsup _{N \rightarrow \infty} \max _{\mathcal{P}} \frac{\chi\left(\Phi_{s}^{(N)}, \mathcal{P}\right)}{N}
$$

where the maximization is performed over all ensemble $\mathcal{P}=\left\{p_{k} ; R_{k}\right\}_{k}$ of $N$ carriers and where

$$
\begin{aligned}
\chi\left(\Phi_{s}^{(N)}, \mathcal{P}\right) \equiv S\left(\Phi_{s}^{(N)}\right. & \left.\left(\sum_{k} p_{k} R_{k}\right)\right) \\
& -\sum_{k} p_{k} S\left(\Phi_{s}^{(N)}\left(R_{k}\right)\right)
\end{aligned}
$$

is the Holevo information [19] associated with $\Phi_{s}^{(N)}$. Kretschmann and Werner have identified a class of maps $\left\{\Phi_{s}^{(n)}\right\}_{n}$ (the forgetful channels [10]) for which the righthand side term of (29) and (31) indeed provide the exact value for $Q_{s}$ and $C_{s}$. Here we will focus only on the limiting cases discussed in Sec. III for which an expression for $Q_{s}$ and $C_{s}$ can be derived without the elegant arguments of Ref. [10]. 
a) The simplest configuration is when the sequence $s$ is such that $\tau_{j} \ll \tau_{E}$ for all $j$. When this happens the maps $\left\{\Phi_{s}^{(n)}\right\}_{n}$ describe a perfect memory channel (14) which allows optimal transfer, ensuring $Q_{s}=C_{s}=\log _{2} D$. Therefore, according to (27) using regular sequences $s$ with $\tau_{j} \ll \tau_{E}$, Alice and Bob can achieve transmission rates equal to

$$
r_{q}(s)=r_{c}(s)=\frac{\log _{2} D}{\tau_{s}} .
$$

b) For memoryless configurations (9), $Q_{s}$ and $C_{s}$ coincide, respectively, with the quantum $Q(\mathcal{N})$ and classical $C(\mathcal{N})$ capacity of the memoryless map $\mathcal{N}$ of Eq. (10). On one hand one has [4],

$$
Q(\mathcal{N})=\lim _{N \rightarrow \infty} \max _{R} \frac{J\left(\mathcal{N}^{\otimes N}, R\right)}{N},
$$

where, as in Eq. (29) the maximization is performed over all density matrices $R$ of $N$ carriers and where $J\left(\mathcal{N}^{\otimes N}, R\right)$ is the coherent information (30) of $\mathcal{N}^{\otimes N}$. On the other hand one has [3],

$$
C(\mathcal{N})=\lim _{N \rightarrow \infty} \max _{\mathcal{P}} \frac{\chi\left(\mathcal{N}^{\otimes N}, \mathcal{P}\right)}{N}
$$

where the maximization is performed over all ensemble $\mathcal{P}=\left\{p_{k} ; R_{k}\right\}_{k}$ of $N$ carriers and where $\chi\left(\mathcal{N}^{\otimes N}, \mathcal{P}\right)$ is the Holevo information (32) associated with $\mathcal{N} \otimes N$. Therefore for regular sequences $s$ with $\tau_{j} \geqslant \tau_{E}$ we get

$$
r_{q}(s)=Q(\mathcal{N}) / \tau_{s}, \quad r_{c}(s)=C(\mathcal{N}) / \tau_{s} .
$$

c) The generalized memoryless configurations (12) can be treated in the same way by replacing the quantities $\tau_{s}^{\prime}, \tau_{s}^{\prime \prime}$ of Eqs. (23) and (26) with the corresponding average first-neighboring group distances and the map $\mathcal{N}$ with the $m$ carriers memoryless map $\mathcal{M}_{s}$ of Eq. (13). In particular, for a generalized memoryless sequences $s$ having constant group lengths $T_{g}=T_{s}$ and constant group separations $\Delta T_{g}=\Delta T_{s}$ for all $g$ one easily verifies the following identities

$$
\begin{aligned}
& r_{q}(s)=Q\left(\mathcal{M}_{s}\right) /\left(T_{s}+\Delta T_{s}\right), \\
& r_{c}(s)=C\left(\mathcal{M}_{s}\right) /\left(T_{s}+\Delta T_{s}\right) .
\end{aligned}
$$

d) Finally consider the noise attenuation protocols of Sec. IIID For the sake of simplicity we will focus on the specific example of Fig. [3] where the results for memoryless configuration applies. In this case the rate is given by

$$
\begin{aligned}
& r_{q}(s)=Q(\overline{\mathcal{N}}) /\left(n \tau+\tau_{E}\right), \\
& r_{c}(s)=C(\overline{\mathcal{N}}) /\left(n \tau+\tau_{E}\right),
\end{aligned}
$$

with $\overline{\mathcal{N}}$ being the map (16) and with $n \tau+\tau_{E}$ being the time intervals which separates two consecutive $A$-carriers.

\section{TRANSMISSION RATE FOR MULTIPLE CHOICE OF THE SEQUENCE}

In this section we analyze the optimal quantum and classical communication rates $R_{q, c}$ achievable in our model when Alice is not restricted to a single given sequence $s$ but instead she has some freedom in selecting the sequence she will use for the signaling.

For the sake of simplicity we will assume the set $\mathcal{S}$ of the allowed sequences to be fully characterized by a single parameter $\tau_{\min }$ which bounds the minimum value for the intervals $\tau_{j}$ of a sequence $s$ of the set. That is $\mathcal{S}=\mathcal{S}\left(\tau_{\min }\right)$ will be the set of all sequences $s$ which satisfy $\tau_{j} \geqslant \tau_{\min }$ for all $j$. The need of constraining the minimum value of the $\tau_{j}$ is fundamental if we want our model to have a non trivial structure (see for instance Sec. IVB and Eq. (46) below). From a more practical point of view the introduction of $\tau_{\min }$ follows from the physical and technological difficulties in producing sequence of ordered signals that might arise in realistic communication scenarios (for instance, too close packed carriers tend to overlap during their propagation, compromising the time ordering of the sequence).

A natural candidate for $R_{q, c}$ is the maximum of the rates $r_{q, c}(s)$ computed over the sequence $s$ of $\mathcal{S}$, i.e.

$$
R_{q, c}^{(1)}\left(\tau_{\min }\right)=\max _{s \in \mathcal{S}} r_{q, c}(s) .
$$

A detailed analysis of $R_{q, c}^{(1)}$ is presented in Appendix $\mathrm{B}$ where it is shown how Eq. (40) simplifies in the case in which $\mathcal{S}$ contains only regular sequences for which Eq. (27) applies. We will see in a moment that for $\tau_{\text {min }} \ll \tau_{E}$ and $\tau_{\text {min }} \geqslant \tau_{E}$ the function $R_{q, c}^{(1)}\left(\tau_{m i n}\right)$ provides indeed the correct values of the achievable rates. For generic $\tau_{\min }$ however we claim that the function $R_{q, c}^{(1)}\left(\tau_{\min }\right)$ does not necessarily tell the whole story about $R_{q, c}$. On the contrary we propose to compute $R_{q, c}$ as follows

$$
\begin{aligned}
& R_{q}\left(\tau_{\min }\right)=\lim _{\epsilon \rightarrow 0} \limsup _{T \rightarrow \infty} \max _{s \in \mathcal{S}} \frac{q_{s}(\epsilon, T)}{T}, \\
& R_{c}\left(\tau_{\min }\right)=\lim _{\epsilon \rightarrow 0} \limsup _{T \rightarrow \infty} \max _{s \in \mathcal{S}} \frac{c_{s}(\epsilon, T)}{T},
\end{aligned}
$$

with $q_{s}(\epsilon, T)$ and $c_{s}(\epsilon, T)$ given in Eqs. (17) and (21). Equations (41) and (42) define proper rates of the communication line of Sec. \} \text { in the sense that, given } \delta > 0 and $\epsilon$ arbitrarily small there is allowed sequence $s \in \mathcal{S}$ which, in the limit of infinite $T$ permit Alice to transfer to Bob at least $\left(R_{q}-\delta\right) T$ qubits with fidelity $>1-\epsilon$.

Since Eq. (40) is obtained from Eqs. (41) and (42) by inverting the order of the maximization over $s$ with the limits in $\epsilon$ and $T$ it follows immediately that $R_{q, c}^{(1)}\left(\tau_{\min }\right)$ is a lower bound for $R_{q, c}\left(\tau_{\min }\right)$ of $\mathcal{S}$, i.e.

$$
R_{q, c}\left(\tau_{\min }\right) \geqslant R_{q, c}^{(1)}\left(\tau_{\min }\right) .
$$

An interesting problem is to understand whether or not the inequality in Eq. (43) can always be replaced with an 
identity. Alternatively one may ask under which conditions on the model parameters (i.e. $U_{j}, \mathcal{F}$ ) the transmission rate of $\mathcal{S}$ can be computed as the maximum of the rates achievable within a specific choice of $s$. In the next section we provide a partial answer to these questions by showing that for $\tau_{\min } \ll \tau_{E}$ and $\tau_{\min } \geqslant \tau_{E}$ the functions $R_{q, c}\left(\tau_{\min }\right)$ and $R_{q, c}^{(1)}\left(\tau_{\min }\right)$ coincide.

\section{A. Bounds and asymptotic behavior}

Even without solving the maximizations of (40), (41) and (42) one expects the resulting expressions $R_{q, c}^{(1)}, R_{q, c}$ will depend upon the interplay between the relaxation time $\tau_{E}$ of LE and the characteristic time $\tau_{\min }$ of $\mathcal{S}$.

A trivial but useful upper bound for $R_{q, c}$ follows by observing that the maximum number $n_{s}(T)$ of carriers that can fit in $\left[0, T\left[\right.\right.$ cannot be greater than $T / \tau_{\min }$ and that $q_{s}(\epsilon, T), c_{s}(\epsilon, T)$ cannot be greater than the $\log _{2}$ of the dimension of $\mathcal{H}(T)$, i.e.

$$
q_{s}(\epsilon, T), c_{s}(\epsilon, T) \leqslant n_{s}(T) \log _{2} D,
$$

with $D$ being the dimension of the Hilbert space of a single carrier. Replacing the above relations in Eqs. (19) and (20) gives

$$
R_{q, c}\left(\tau_{\min }\right) \leqslant \frac{\log _{2} D}{\tau_{\min }},
$$

for all $\tau_{\text {min }}$. From Sec. IVB it follows that this bound is achievable at least if $\mathcal{S}$ is such that $\tau_{\min } \ll \tau_{E}$. In this case in fact the sequence $s_{0}$ with $\tau_{j}=\tau_{\min }$ for all $j$ allows for carriers that reliably transfer $\log _{2} D$ qubits of information each. Therefore from (40) and (43) we get

$$
R_{q, c}\left(\tau_{\min }\right)=\left.R_{q, c}^{(1)}\left(\tau_{\min }\right)\right|_{\tau_{\min } \ll \tau_{E}} \simeq \frac{\log _{2} D}{\tau_{\min }},
$$

which shows that the rates diverge for $\tau_{\min } \rightarrow 0$. An explicit expression can also be determined for $\tau_{\min }$ greater than $\tau_{E}$. In fact, according to Sec. IIIA in this case all the allowed sequences $s$ yields the same memoryless mapping $\mathcal{N}^{\otimes n(T)}$. Thus the maximization with respect to $s$ becomes a simple optimization with respect to the average time intervals $\tau_{s}$ and one gets,

$$
\begin{aligned}
& R_{q}\left(\tau_{\text {min }}\right)=\left.R_{q}^{(1)}\left(\tau_{\text {min }}\right)\right|_{\tau_{\text {min }} \geqslant \tau_{E}}=Q(\mathcal{N}) / \tau_{\text {min }}, \\
& R_{c}\left(\tau_{\text {min }}\right)=\left.R_{c}^{(1)}\left(\tau_{\text {min }}\right)\right|_{\tau_{\text {min }} \geqslant \tau_{E}}=C(\mathcal{N}) / \tau_{\text {min }}
\end{aligned}
$$

with $Q(\mathcal{N})$ and $C(\mathcal{N})$ the capacities of Eqs. (34) and (355), respectively.

For intermediate value of $\tau_{\min }$ a lower bound for $R_{q, c}^{(1)}$, and thus for $R_{q, c}$, can be obtained for instance by focusing on the generalized memoryless configuration (see Eq. (B1) or by considering the noise attenuation strategies. In this last case it is simpler to consider only the

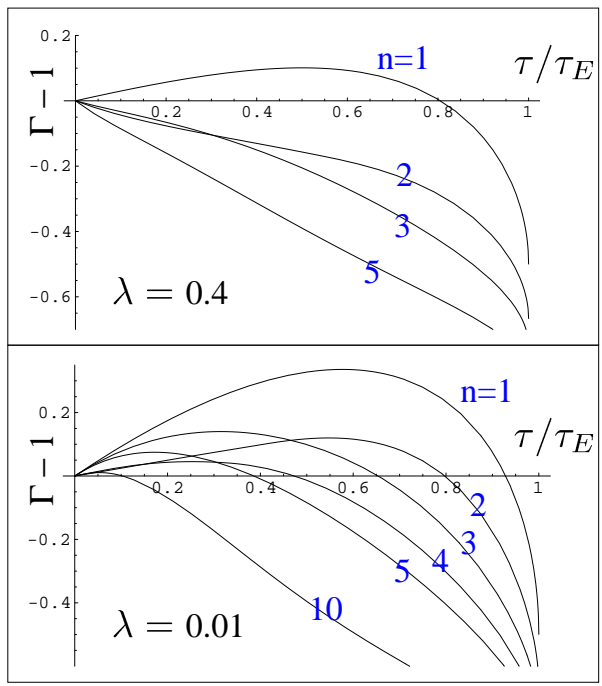

FIG. 4: Plot of the ratio $\Gamma$ of Eq. (56) as a function of the dimensionaless parameter $\tau / \tau_{E}$, for different values of the $n$ and for different values of the environment-carriers coupling constant $\lambda$. In the strong coupling regime $\lambda \sim 0$, the attenuation noise protocol provides a significative improvement of the transmission rate. For instance for $\lambda=0.01, r$ reaches the maximum value of $\sim 1.3$ for $n=1$ and $\tau \sim \tau_{E} / 2$.

configurations described in Fig. 3 and maximizing the rates (39) with respect to the free parameters $\tau \geqslant \tau_{\min }$ and $n \geqslant 1$, e.g.

$$
\begin{aligned}
& R_{q}^{(1)}\left(\tau_{\text {min }}\right) \geqslant \sup _{\substack{\tau \geqslant \tau_{\min } \\
n \geqslant 1}} \frac{Q(\overline{\mathcal{N}})}{n \tau+\tau_{E}}, \\
& R_{c}^{(1)}\left(\tau_{\text {min }}\right) \geqslant \sup _{\substack{\tau \geqslant \tau_{\text {min }} \\
n \geqslant 1}} \frac{C(\overline{\mathcal{N}})}{n \tau+\tau_{E}} .
\end{aligned}
$$

\section{AN EXAMPLE WITH QUBITS}

In this section we analyze an example of dynamical model for memory channels where both the information carriers $\mathrm{C}_{j}$ and the local environment LE are qubits. In this context we will make a comparison between the noise attenuation protocol of Sec. IIID and the memoryless configuration.

We will assume the carrier-LE interaction $U_{j}$ of Eq. (31) to be to a control-unitary such that when the carrier is in $|0\rangle_{C_{j}}$ nothing happens to LE, while when $\mathrm{C}_{j}$ is in $|1\rangle_{C_{j}}$ the environment undergoes to the transformation

$$
\Theta(\lambda) \equiv\left(\begin{array}{cc}
\sqrt{\lambda} & \sqrt{1-\lambda} \\
\sqrt{1-\lambda} & -\sqrt{\lambda}
\end{array}\right),
$$

with $\lambda \in[0,1]$ being a parameter which measures the "intensity" of the coupling (with low coupling corresponding to $\lambda \sim 1$ and high coupling corresponding to 
$\lambda \sim 0$ ). Moreover we will assume the relaxation process $\mathcal{F}=\left\{\mathcal{E}_{\tau}\right\}_{\tau}$ acting on LE to be described by amplitude damping maps [1] which takes the state $|1\rangle_{L E}$ to $|0\rangle_{L E}$ with probability $1-\eta(\tau)$ where $\eta(\tau) \in[0,1]$ is a non increasing function of $\tau$ with characteristic time $\tau_{E}$, i.e.

$$
\begin{aligned}
& \mathcal{E}_{\tau}\left(|0\rangle_{L E}\langle 0|\right)=|0\rangle_{L E}\langle 0| \\
& \mathcal{E}_{\tau}\left(|1\rangle_{L E}\langle 1|\right)=\eta(\tau)|1\rangle_{L E}\langle 1|+(1-\eta(\tau))| 0\rangle_{L E}\langle 0| \\
& \mathcal{E}_{\tau}\left(|0\rangle_{L E}\langle 1|\right)=\sqrt{\eta(\tau)}|0\rangle_{L E}\langle 1| .
\end{aligned}
$$

In this example the stationary state $\sigma_{0}$ of LE is hence $|0\rangle_{L E}$. The parameterization of the memory effect is given by $\eta(\tau)$, with $\eta=0$ corresponding to the memoryless case (fast environment relaxation) and $\eta=1$ corresponding to perfect memory case (no environment relaxation). In order to have a well defined threshold between memoryless and memory configuration, in the following we will assume

$$
\eta(\tau)= \begin{cases}1-\tau / \tau_{E} & \text { for } \tau<\tau_{E} \\ 0 & \text { for } \tau \geqslant \tau_{E}\end{cases}
$$

Under the above conditions, it is possible to show that both the map $\mathcal{N}$ of the memoryless case and the map $\overline{\mathcal{N}}$ (16) of the noise attenuation protocol correspond to a phase damping channel $\mathcal{P}_{g}$ where the coherence terms of the input qubit $\rho$ are degraded by a positive factor $g \leqslant 1$, i.e. 1]

$$
\begin{aligned}
& \mathcal{P}_{g}\left(|\kappa\rangle_{C}\langle\kappa|\right)=|\kappa\rangle_{C}\langle\kappa| \quad \text { for } \kappa=0,1 \\
& \mathcal{P}_{g}\left(|0\rangle_{C}\langle 1|\right)=g|0\rangle_{C}\langle 1| .
\end{aligned}
$$

In particular Eq. (10) gives $\mathcal{N}=\mathcal{P}_{g_{0}}$ with $g_{0}=\sqrt{\lambda}$. On the other hand, Eq. (16) gives $\overline{\mathcal{N}}=\mathcal{P}_{\bar{g}}$ where $\bar{g}$ is a complicated expression (C10) of $\lambda$ and of the parameters $\rho_{0}$, $n$ and $\tau$ (see Appendix C for details). By appropriately selecting the values of the above quantities one can make the make $\overline{\mathcal{N}}$ less noisy than $\mathcal{N}$ by having $\bar{g}>g_{0}$. To see if this corresponds to an increase in the transmission rates $r_{q, c}(s)$ we can use the results of Sec. IVB In the case of the phase damping channels $\mathcal{P}_{g}$ the capacities $Q\left(\mathcal{P}_{g}\right)$ and $C\left(\mathcal{P}_{g}\right)$ of Eqs. (34) and (35) can be explicitly computed. For instance since here the noise does not affect the populations associated with the computational basis, the classical capacity of the phase damping channel (53) is optimal for all values of $g$, i.e. $C\left(\mathcal{P}_{g}\right)=1$. Hence from Eqs. (36) and (39) we get

$$
r_{c}\left(s_{0}\right)=1 / \tau_{E} \geqslant 1 /\left(n \tau+\tau_{E}\right)=\bar{r}_{c},
$$

where $s_{0}$ is the memoryless sequence with uniform interval $\tau_{j}=\tau_{E}$ and $\bar{r}_{c}$ is the classical rate of the noise attenuation protocol of Fig. [3] Equation (54) shows that, in the specific example considered here, the noise attenuation protocol does not improve the classical rate of the communication line with respect to the memoryless case. On the other hand the quantum capacity of a phase damping channel (53) is equal to [20]

$$
Q\left(\mathcal{P}_{g}\right)=1-H_{2}(1 / 2+g / 2),
$$

where $H_{2}(x)=-x \log _{2} x-(1-x) \log _{2}(1-x)$ is the binary entropy function. In this case, higher values of $g$ corresponds to higher $Q\left(\mathcal{P}_{g}\right)$ and the rate $\bar{r}_{q}$ of the noise attenuation protocol can be higher than the rate $r_{q}\left(s_{0}\right)$ of the memoryless case. To see this we studied the ratio

$$
\Gamma=\frac{\bar{r}_{q}}{r_{q}\left(s_{0}\right)}=\frac{\tau_{E}}{n \tau+\tau_{E}} \frac{1-H_{2}(1 / 2+\bar{g} / 2)}{1-H_{2}\left(1 / 2+g_{0} / 2\right)},
$$

as a function of the variable $\tau / \tau_{E}$ and for for different values of $n$ and $\lambda$. [Here $\bar{g}$ has been optimized with respect to the no-carrying signal $\left.\rho_{0}\right]$. The results have been plotted in Fig. 4 which shows that in the strong coupling limit $\lambda \sim 0$ one can have an appreciable increase of $\Gamma$ for $\tau \sim \tau_{E} / 2$ and with $n$ of the order of 5 .

\section{CONCLUSION}

We have introduced a communication model where memory effects arise from the interaction between the information carriers with the channel environment. Different memory effects can be simulated by varying the time intervals at which the carriers are produced by the sender of the message. The information rates of the model have been defined and computed in some extremal cases.

\section{APPENDIX A}

In this appendix we show how a Markovian correlated noise [6, 9, 10] can be derived from the mapping (8) by properly choosing the transformation $\mathcal{E}_{\tau_{j}}$.

Consider the case in which for sufficiently big $\tau$ the map $\mathcal{E}_{\tau}$ describes a decoherent process of LE where, given $\left\{|\ell\rangle_{L E}\right\}$ an orthonormal basis of $\mathcal{H}_{L E}$, one has

$$
\mathcal{E}_{\tau}\left(|\ell\rangle_{L E}\left\langle\ell^{\prime}\right|\right)=\delta_{\ell, \ell^{\prime}}\left|\psi_{\ell}(\tau)\right\rangle_{L E}\left\langle\psi_{\ell}(\tau)\right|,
$$

with the vectors $\left\{\left|\psi_{\ell}(\tau)\right\rangle_{L E}\right\}_{\ell}$ being not necessarily orthogonal, and $\delta_{\ell, \ell^{\prime}}$ being the Kronecker delta. The condition (5) can then be satisfied by identifying $\sigma_{0}$ with one element of the selected basis (say $\left|\ell_{0}\right\rangle_{L E}$ ), and imposing $\left|\psi_{\ell}\left(\tau \geqslant \tau_{E}\right)\right\rangle_{L E}=\left|\ell_{0}\right\rangle_{L E}$ for all $\ell$. In this case the mapping (8) can be expressed in terms of the operators

$$
\begin{aligned}
A_{\ell_{1}} & \equiv{ }_{L E}\left\langle\ell_{1}\left|U_{1}\right| \ell_{0}\right\rangle_{L E} \\
A_{\ell_{j+1}, \ell_{j}} & \equiv{ }_{L E}\left\langle\ell_{j+1}\left|U_{j+1}\right| \psi_{\ell_{j}}\left(\tau_{j}\right)\right\rangle_{L E},
\end{aligned}
$$

which act, respectively, on the Hilbert space $\mathcal{H}_{C_{1}}$ and $\mathcal{H}_{C_{j+1}}$ for $j=1, \cdots, n-1$. They allow us to define the probability distribution

$$
p_{\ell_{1}}^{(1)} \equiv \operatorname{Tr}_{C_{1}}\left\{A_{\ell_{1}}^{\dagger} A_{\ell_{1}}\right\}
$$

and the conditional probabilities

$$
p_{\ell_{j+1} \mid \ell_{j}}^{(j+1)} \equiv \operatorname{Tr}_{C_{j+1}}\left\{A_{\ell_{j+1}, \ell_{j}}^{\dagger} A_{\ell_{j+1}, \ell_{j}}\right\} .
$$


which satisfies the normalization conditions $\sum_{\ell_{j+1}} p_{\ell_{j+1} \mid \ell_{j}}^{(j+1)}=1$ and $\sum_{\ell_{j}} p_{\ell_{j+1} \mid \ell_{j}}^{(j+1)}<1$. Using these quantities Eq. (8) can be finally expressed in compact Markovian form,

$$
\begin{gathered}
\Phi_{s}^{(n)}(R)=\sum_{\ell_{1}, \cdots, \ell_{n}} p_{\ell_{1}}^{(1)} p_{\ell_{2} \mid \ell_{1}}^{(2)} \cdots p_{\ell_{n} \mid \ell_{n-1}}^{(n)} \\
\times M_{\ell_{n}, \ell_{n-1}} \cdots M_{\ell_{2}, \ell_{1}} M_{\ell_{1}} R M_{\ell_{1}}^{\dagger} M_{\ell_{2}, \ell_{1}}^{\dagger} \cdots M_{\ell_{n}-1, \ell_{n}}^{\dagger}
\end{gathered}
$$

with $M_{\ell_{1}} \equiv A_{\ell_{1}} / \sqrt{p_{\ell_{1}}^{(1)}}$ and

$$
M_{\ell_{j+1}, \ell_{j}} \equiv A_{\ell_{j+1}, \ell_{j}} / \sqrt{p_{\ell_{j+1} \mid \ell_{j}}^{(j+1)}}
$$

\section{APPENDIX B}

In this section we analyze $R_{q, c}^{(1)}$ showing that, if the set $\mathcal{S}$ contains only regular sequences, then the maximization of Eq. (40) can be solved by focusing on the generalized memoryless configurations.

Consider the subset $\mathcal{S}_{0}$ of the sequence $s \in \mathcal{S}$ which correspond to the uniform generalized memoryless configurations of Sec. IIIB characterized by constant group distance $\Delta T_{s}=\max \left\{\tau_{\min }, \tau_{E}\right\}$. Since $\mathcal{S}_{0}$ is a proper subset of $\mathcal{S}$ we have

$$
\begin{aligned}
R_{q}^{(1)}\left(\tau_{\min }\right) & \geqslant \max _{s \in \mathcal{S}_{0}} r_{q}(s) \\
& =\max _{s \in \mathcal{S}_{0}} \frac{Q\left(\mathcal{M}_{s}\right)}{T_{s}+\max \left\{\tau_{\min }, \tau_{E}\right\}},
\end{aligned}
$$

where we used Eq. (37) to express $r_{q}(s)$. Now, given $s \in \mathcal{S}$ from Eqs. (22) and (29) one gets

$$
\begin{aligned}
r_{q}(s) & \leqslant\left(1 / \tau_{s}^{\prime}\right) \limsup _{N \rightarrow \infty} \max _{R}\left\{J\left(\Phi_{s}^{(N)}, R\right) / N\right\} \\
& \leqslant\left(1 / \tau_{s}^{\prime}\right) \limsup _{N \rightarrow \infty}\left\{\sup _{k \geqslant 1} \max _{R^{\prime}} \frac{J\left(\left[\Phi_{s}^{(N)}\right]^{\otimes k}, R^{\prime}\right)}{k N}\right\} \\
& =\left(1 / \tau_{s}^{\prime}\right) \limsup _{N \rightarrow \infty}\left\{\lim _{k \rightarrow \infty} \max _{R^{\prime}} \frac{J\left(\left[\Phi_{s}^{(N)}\right]^{\otimes k}, R^{\prime}\right)}{k N}\right\} \\
& =\left(1 / \tau_{s}^{\prime}\right) \limsup _{N \rightarrow \infty}\left\{\frac{Q\left(\Phi_{s}^{(N)}\right)}{N}\right\},
\end{aligned}
$$

where in the second and in the third line the maximization is performed over the density matrix $R^{\prime}$ of $k \times N$ carriers, $\left[\Phi_{s}^{(N)}\right]^{\otimes k}$ are $k$ copies of the map $\Phi_{s}^{(N)}$, and $Q\left(\Phi_{s}^{(N)}\right)$ is the memoryless quantum capacity (34) of the $\operatorname{map} \Phi_{s}^{(N)}$. The second inequality is trivial: it follows from the fact that $\max _{R} J\left(\Phi_{s}^{(N)}, R\right) / N$ can be obtained from $\max _{R^{\prime}} J\left(\left[\Phi_{s}^{(N)}\right]^{\otimes k}, R^{\prime}\right) /(k N)$ for $k=1$. The identity on the third line instead is a consequence of the fact that $\max _{R^{\prime}} J\left(\left[\Phi_{s}^{(N)}\right]^{\otimes k}, R^{\prime}\right) /(k N)$ achieves its maximum for $k \rightarrow \infty$. We can further simplify the above expression by introducing the time interval $T_{s}(N-1)=\sum_{j=1}^{N-1} \tau_{j}$ associated with the first $N-1$ carriers of the sequence $s$ and noticing that

$$
\limsup _{N \rightarrow \infty} \frac{T_{s}(N-1)}{N}=\tau_{s}^{\prime \prime},
$$

with $\tau_{s}^{\prime \prime}$ defined as in Eq. (26). Using this result, from Eq. (B2) we get

$$
\begin{aligned}
r_{q}(s) \leqslant & \limsup _{N \rightarrow \infty} \frac{T_{s}(N-1)+\max \left\{\tau_{\min }, \tau_{E}\right\}}{N \tau_{s}^{\prime}} \\
& \times \limsup _{N \rightarrow \infty} \frac{Q\left(\Phi_{s}^{(N)}\right)}{T_{s}(N-1)+\max \left\{\tau_{\min }, \tau_{E}\right\}} \\
\leqslant & \frac{\tau_{s}^{\prime \prime}}{\tau_{s}^{\prime}} \sup _{N} \frac{Q\left(\Phi_{s}^{(N)}\right)}{T_{s}(N-1)+\max \left\{\tau_{\min }, \tau_{E}\right\}} \\
\leqslant & \frac{\tau_{s}^{\prime \prime}}{\tau_{s}^{\prime}} \sup _{s \in \mathcal{S}_{0}} \frac{Q\left(\mathcal{M}_{s}\right)}{T_{s}+\max \left\{\tau_{\min }, \tau_{E}\right\}} .
\end{aligned}
$$

The ratio $\tau_{s}^{\prime \prime} / \tau_{s}^{\prime}$ is always greater than or equal to one. However, if the set $\mathcal{S}$ includes only sequences which are regular, than for all $s$ we have $\tau_{s}^{\prime}=\tau_{s}^{\prime \prime}$. In this case the bounds of Eqs. (B1) and (B4) coincides yielding

$$
R_{q}^{(1)}\left(\tau_{\min }\right)=\max _{s \in \mathcal{S}_{0}} \frac{Q\left(\mathcal{M}_{s}\right)}{T_{s}+\max \left\{\tau_{\min }, \tau_{E}\right\}} .
$$

The same derivation applies also for the classical rate $R_{c}^{(1)}$. In this case one can show that if $\mathcal{S}$ contains only regular sequence then,

$$
R_{c}^{(1)}\left(\tau_{\min }\right)=\max _{s \in \mathcal{S}_{0}} \frac{C\left(\mathcal{M}_{s}\right)}{T_{s}+\max \left\{\tau_{\min }, \tau_{E}\right\}} .
$$

\section{a. Asymptotic limit}

It is interesting to note that the above expressions give the correct asymptotic values of Sec. $\mathrm{VA}$ For instance for $\tau_{\text {min }} \geqslant \tau_{E}$ we have $\mathcal{M}_{s}=\mathcal{N}^{\otimes m}$ where $m$ is the number of carriers contained in each group of the sequence and $\mathcal{N}$ is the memoryless map (9). Given $s \in \mathcal{S}_{0}$ this yields

$$
\frac{Q\left(\mathcal{M}_{s}\right)}{T_{s}+\max \left\{\tau_{\min }, \tau_{E}\right\}}=\frac{m Q(\mathcal{N})}{T_{s}+\tau_{\min }} \leqslant \frac{Q(\mathcal{N})}{\tau_{\min }}
$$

where we used the additivity property $Q\left(\mathcal{N}^{\otimes m}\right)=$ $m Q(\mathcal{N})$ of memoryless channels and the fact that group length (111) is always greater or equal to $(m-1) \tau_{\text {min }}$. Equation (47) finally follows by noticing that the rate $Q(\mathcal{N}) / \tau_{\min }$ is achieved by the sequence of $\mathcal{S}_{0}$ with $\tau_{g, \ell}=$ $\tau_{\min }$ for all $g$ and $\ell$.

The limit (46) instead follows by noticing that the rate $\log _{2} D / \tau_{\text {min }}$ can be obtained from the set $\mathcal{S}_{0}$ by using $\tau_{g, \ell}=\tau_{\min }$ for all $\ell=1, \cdots, m-1$ in the limit of large group, i.e. $m \rightarrow \infty$. In this case in fact 
$\mathcal{M}_{s}$ is a tensor product of perfect memory channels and $T_{s}=(m-1) \tau_{m i n}$, so that

$$
\begin{aligned}
\frac{Q\left(\mathcal{M}_{s}\right)}{T_{s}+\max \left\{\tau_{\min }, \tau_{E}\right\}} & =\frac{m \log _{2} D}{(m-1) \tau_{\min }+\tau_{E}} \\
& \rightarrow \frac{\log _{2} D}{\tau_{\min }}
\end{aligned}
$$

\section{APPENDIX C}

To characterize the modified map of $\overline{\mathcal{N}}$ we first solve the system (15) by using the following parameterization for the density matrices element of $\sigma_{j}$ in the canonical basis $\left\{|0\rangle_{L E},|1\rangle_{L E}\right\}$,

$$
\sigma_{j} \equiv\left(\begin{array}{cc}
1-z_{j} & x_{j}+i y_{j} \\
x_{j}-i y_{j} & z_{j}
\end{array}\right)
$$

with $z_{j} \in[0,1]$ and $x_{j}, y_{j}$ real for all $j=0,1, \cdots, n$. The resulting recursive equation can be simplified by introducing the column vectors

$$
\begin{gathered}
\vec{v}_{j} \equiv\left(\eta^{-1 / 4} z_{j}, x_{j}\right)^{T} \\
\vec{w} \equiv(1-p)\left(\eta^{3 / 4}(1-\lambda), \eta^{1 / 4} \sqrt{\lambda(1-\lambda)}\right)^{T}
\end{gathered}
$$

and the $2 \times 2$ Hermitian matrix

$$
A \equiv(1-p)\left[\begin{array}{cc}
\eta\left(\frac{p}{1-p}-1+2 \lambda\right) & -2 \eta^{3 / 4} \sqrt{\lambda(1-\lambda)} \\
-2 \eta^{3 / 4} \sqrt{\lambda(1-\lambda)} & \sqrt{\eta}\left(\frac{p}{1-p}+1-2 \lambda\right)
\end{array}\right]
$$

where $\eta$ stands for $\eta(\tau)$ and $p$ is the population associated with the $|0\rangle_{C}$ component of the no-carrying message state $\rho_{0}$. In this notation Eq. (15) gives the following uncoupled equations

$$
\begin{aligned}
& y_{j+1}=\sqrt{\eta}(2 p-1) y_{j} \\
& \vec{v}_{j+1}=A \cdot \vec{v}_{j}+\vec{w}
\end{aligned}
$$

which can be solved analytically. In particular, imposing the initial condition $\sigma_{0}=|0\rangle_{L E}\langle 0|$ (i.e. $x_{0}=y_{0}=z_{0}=$ 0 ) the first one gives $y_{j}=0$ for all $j$. The solution of (C3 instead can be obtained in terms of the eigenvalues $\lambda_{ \pm}$of $A$ and their corresponding eigenvectors $\left(\alpha_{ \pm}, \beta_{ \pm}\right)^{T}$. Explicitly the eigenvalues of $A$ are

$$
\begin{aligned}
\lambda_{ \pm}= & \frac{\sqrt{\eta}}{2}[(1+\sqrt{\eta}) p \\
& +(1-p)(1-\sqrt{\eta})(1-2 \lambda) \pm \Delta],
\end{aligned}
$$

with

$$
\begin{aligned}
\Delta= & \{4 \sqrt{\eta}(1-2 p) \\
& \left.+[(1+\sqrt{\eta}) p+(1-p)(1-\sqrt{\eta})(1-2 \lambda)]^{2}\right\}^{1 / 2} .
\end{aligned}
$$

The corresponding eigenvectors $\left(\alpha_{ \pm}, \beta_{ \pm}\right)$have instead the following components

$\alpha_{ \pm}=\eta^{1 / 4}(1-p) \sqrt{\lambda(1-\lambda)} / N_{ \pm}$,

$\beta_{ \pm}=[(\sqrt{\eta}-1) p-(1-p)(1-2 \lambda)(1+\sqrt{\eta}) \mp \Delta] / N_{ \pm}$, with the normalization coefficient

$$
\begin{gathered}
N_{ \pm}=\left\{16(1-p)^{2} \sqrt{\eta} \lambda(1-\lambda)\right. \\
\left.+[(\sqrt{\eta}-1) p-(1-p)(1-2 \lambda)(1+\sqrt{\lambda}) \mp \Delta]^{2}\right\}^{1 / 2} .
\end{gathered}
$$

In particular, for $\left|\lambda_{ \pm}\right|<1$ one has 21]

$$
\vec{v}_{j}=A^{j} \cdot \vec{v}_{0}+\sum_{k=0}^{j-1} A^{k} \cdot \vec{w}=\frac{\mathbb{1}-A^{j}}{\mathbb{1}-A} \cdot \vec{w}
$$

and thus

$$
\begin{aligned}
& z_{j}=\eta^{3 / 4}(1-p)\left[\eta^{1 / 4}(1-\lambda) u^{(j)}+\sqrt{\lambda(1-\lambda)} t^{(j)}\right] \\
& x_{j}=\eta^{1 / 2}(1-p)\left[\eta^{1 / 4}(1-\lambda) t^{(j)}+\sqrt{\lambda(1-\lambda)} v^{(j)}\right],
\end{aligned}
$$

where $u^{(j)}=\xi_{+}^{(j)} \alpha_{+}^{2}+\xi_{-}^{(j)} \alpha_{-}^{2}, v^{(j)}=\xi_{+}^{(j)} \beta_{+}^{2}+\xi_{-}^{(j)} \beta_{-}^{2}$, and $t^{(j)}=\xi_{+}^{(j)} \alpha_{+} \beta_{+}+\xi_{-}^{(j)} \alpha_{-} \beta_{-}$with

$$
\xi_{ \pm}^{(j)}=\frac{1-\left(\lambda_{ \pm}\right)^{j}}{1-\lambda_{ \pm}}
$$

Setting $j=n$ and replacing the above expressions into (C1) we obtain the modified state of LE $\sigma_{n}$ after $n$ successive interactions with $\rho_{0}$. Using the definition (16) one verifies that $\overline{\mathcal{N}}$ is a phase damping channel (53) characterized by a damping factor

$$
\bar{g}=\sqrt{\lambda}-2\left(\sqrt{\lambda} z_{n}-\sqrt{1-\lambda} x_{n}\right)
$$

\section{ACKNOWLEDGMENTS}

I would like to thank Rosario Fazio for remarks and suggestions: without his encouragement this work would never been completed. Moreover I would like to thank Chiara Macchiavello and Massimo Palma for their comments and discussions. In particular, thank to Massimo for pointing out Refs. [12].
[1] M. A. Nielsen and I. L. Chuang, Quantum Computation and Quantum Information (Cambridge University Press, Cambridge 2000).

[2] C. H. Bennett and P. W. Shor, IEEE Trans. Inf. Theory
44, 2724 (1998).

[3] A. S. Holevo, IEEE Trans. Inf. Theory 44, 269 (1998); P. Hausladen, R. Jozsa, B. Schumacher, M. Westmoreland and W. K. Wootters, Phys. Rev. A 54, 1869 (1996); B. 
Schumacher and M. D. Westmoreland, Phys. Rev. A 56, 131 (1997).

[4] S. Lloyd, Phys. Rev. A 55, 1613 (1997); H. Barnum, M. A. Nielsen, and B. Schumacher, Phys. Rev. A 57, 4153 (1998); I. Devetak, IEEE Trans. Inform. Theory 51, 44 (2005).

[5] C.H. Bennett, P. W. Shor, J. A. Smolin, and A. V. Thapliyal, IEEE Trans. Inf. Theory 48, 2637 (2002).

[6] C. Macchiavello and G. M. Palma, Phys. Rev. A 65, 050301(R) (2002); C. Macchiavello, G. M. Palma, and S. Virmani, Phys. Rev. A 69, 010303(R) (2004).

[7] V. Giovannetti and S. Mancini, Phys. Rev. A 71, 062304 (2005). G. Ruggeri, G. Soliani, V. Giovannetti, and S. Mancini, Europhys. Lett., 70, 719 (2005).

[8] N. J. Cerf, J. Clavareau, C. Macchiavello, and J. Roland, Phys. Rev. A accepted for publication, eprint quant-ph/0412089

[9] G. Bowen and S. Mancini, Phys. Rev. A 69, 012306 (2004).

[10] D. Kretschmann and R. F. Werner, eprint quant-ph/0502106

[11] H. P. Breuer and F. Petruccione, The theory of open quantum systems (Oxford University Press, Oxford 2002).

[12] V. Scarani, M. Ziman, P. Štelmachovič, N. Gisin, and V. Bužek, Phys. Rev. Lett. 88, 097905 (2002); M. Ziman, P. Štelmachovič, V. Bužek, M. Hillery, V. Scarani, and
N. Gisin Phys. Rev. A, 65, 042105 (2002).

[13] The integer $n_{s}(T)$ is uniquely defined by the inequalities

$$
\sum_{j=1}^{n_{s}(T)-1} \tau_{j} \leqslant T<\sum_{j=1}^{n_{s}(T)} \tau_{j} .
$$

[14] The presence of $\lim \sup _{T \rightarrow \infty} f(T)=\lim _{T \rightarrow \infty} \sup _{t \geqslant T} f(t)$ in place of a regular limit over $T$ in Eq. (19) stems from the fact that one is considering the maximum over all possible rate achievable. Analogous definitions applies in the case of channel capacities of memoryless channels (see for instance Refs. [5, 10, 16, 17]).

[15] The inequalities (22) and (25) do not apply, respectively, in the pathological situation where $\left(\tau_{s}^{\prime}, Q_{s}\right)=(0,0)$ and $\left(\tau_{s}^{\prime \prime}, Q_{s}\right)=(0,0)$. These cases are hence excluded from the analysis.

[16] H. Barnum, E. Knill, and M. A. Nielsen, IEEE Trans. Inf. Theory 46, 1317 (2000).

[17] D. Kretschmann and R. F. Werner, New Journal of Physics 6, 26 (2004).

[18] B. Schumacher and M. A. Nielsen, Phys. Rev. A 54, 2629 (1996).

[19] A. S. Holevo, Probl. Inf. Trans. 9, 177 (1973).

[20] I. Devetak and P. W. Shor, eprint quant-ph/0311131

[21] The case in which one or both the eigenvalues of $A$ have unitary modulus can be treated analogously. 